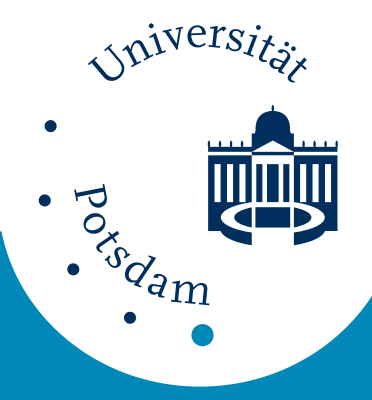

Universität Potsda m

Egbert Zienicke, Norbert Seehafer, Fred Feudel

Bifurcations in two-dimensional

Rayleigh-Bénard convection

NLD Preprints ; 42 


\title{
Bifurcations in two-dimensional Rayleigh-Bénard convection
}

\author{
E. Zienicke, ${ }^{1}$ N. Seehafer ${ }^{2}$ and F. Feudel ${ }^{2}$ \\ ${ }^{1}$ Observatoire de la Côte d'Azur, CNRS URA 1362, B.P. 229, F 06304 Nice Cedex 4, France \\ ${ }^{2}$ Institut für Physik, Universität Potsdam, PF 601553, D-14415 Potsdam, Germany
}

(August 27, 1997)

Two-dimensional bouyancy-driven convection in a horizontal fluid layer with stress-free boundary conditions at top and bottom and periodic boundary conditions in the horizontal direction is investigated by means of numerical simulation and bifurcation-analysis techniques. As the bouyancy forces increase, the primary stationary and symmetric convection rolls undergoe successive Hopf bifurcations, bifurcations to traveling waves, and phase lockings. We pay attention to symmetry breaking and its connection with the generation of large-scale horizontal flows. Calculations of Lyapunov exponents indicate that at a Rayleigh number of $2.3 \cdot 10^{5}$ no temporal chaos is reached yet but the system moves nonchaotically on a 4torus in phase space.

47.20.Ky, 47.20.Bp, 47.54.+r

\section{INTRODUCTION}

Bouyancy-driven convection in a fluid layer heated from below, known as Rayleigh-Bénard convection, is one of the best-studied hydrodynamical systems [1-5]. Apart from its many applications, notably in meteorology, geophysics and astrophysics, it has become a paradigm of instabilities, bifurcations, structure formation and chaotic bchavior in fluid dynamics as well as in physical systems in general.

The simplest, but very influential model to study the nonlinear dynamics of this system was the one derived by Lorenz [6], which consisted of only three ordinary differential equations, resulting from a hard truncation of a Fourier expansion of the equations of two-dimensional convection. The most important property of this model was the appearance of chaos in certain intervals of the system parameters. An extension of the Lorenz model to 14 modes was studied by Curry [7], who also found chaos. By contrast, no chaos was found in high-resolution twodimensional calculations with stress-free boundary conditions at top and bottom by Curry et al. [8]. These authors concluded that the appearance of chaos in the low-order [6,7] models was a result of using an insufficient number of modes. However, for computational speed-up Curry et al. had imposed special symmetries, thus restricting the dynamics to an invariant subspace of symmetric solutions and possibly suppressing symmetrybreaking bifurcations. In more recent calculations by Prat et al. $[9,10]$ for the case of no-slip boundary conditions and no restrictions by symmetry, chaotic behavior was again found. A particular issue addressed by Prat et al. was the generation of large-scale horizontal flows and their vertical profiles (e.g., symmetric or antisymmetric to the horizontal midline).

In the present paper we study the bifurcations in twodimensional Rayleigh-Bénard convection with stress-free boundary conditions and no symmetry imposed. For a variety of astrophysical and geophysical applications, for instance the convective layers in stars like the sun or convection in the earth mantle, stress-free conditions are probably a better choice than no-slip conditions, which are realized in most laboratory experiments. Stress-free boundary conditions are difficult to control in the laboratory, but an experiment with these conditions has been reported [11]. Possibilities to approximately realize twodimensionality are discussed in Sec. VI.

In Sec. II we introduce the governing equations, while in Sec. III the symmetries of the system and their connection with the possible occurence of large-scale flows are discussed. Then in Secs. IV and V we describe the bifurcations found. Sec. VI, finally, gives a discussion of our results.

\section{EQUATIONS}

We investigate bouyancy-driven convection in a twodimensional fluid layer of thickness $d$ heated from below. Using the Oberbeck-Boussinesq approximation, which is correct for a wide variety of fluids [1,5], one has to solve the partial differential equations

$$
\begin{aligned}
\nabla \cdot \mathbf{v} & =0 \\
\frac{\partial \mathbf{v}}{\partial t}+(\mathbf{v} \cdot \nabla) \mathbf{v} & =-\nabla p+\mathcal{P} \triangle \mathbf{v}+\mathcal{P} \mathcal{R} \theta \mathbf{e}_{y} \\
\frac{\partial \theta}{\partial t}+\mathbf{v} \cdot \nabla \theta & =v_{y}+\triangle \theta
\end{aligned}
$$

where $\mathbf{v}$ is the fluid velocity, while $p$ and $\theta$ denote the deviations of pressure and temperature from their values in the quiescent ground state with pure heat conduction. We use rectangular Cartesian coordinates $x$ and $y$, with the $x$ axis in the horizontal and the $y$ axis in the vertical direction parallel to the gravitational force; $\mathbf{e}_{y}$ is the unit vector in the vertical direction. Equations (1)-(3) are given in dimensionless form, with the thickness $d$ of the fluid layer as the unit of length, $\kappa / d^{2}$, where $\kappa$ is the thermal diffusivity, as the unit of time, and the temperature difference $\delta T$ between lower and upper boundaries of the fluid layer as the unit of temperature. There are 
two parameters, the Prandtl number $\mathcal{P}$ and the Rayleigh number $\mathcal{R}$, defined by

$$
\mathcal{P}=\frac{\nu}{\kappa}, \quad \mathcal{R}=\frac{\alpha g d^{3}}{\nu \kappa} \delta T,
$$

where $\nu$ is the kinematic viscosity, $\alpha$ the volumetric expansion coefficient, and $g$ the gravitational acceleration. $\mathcal{R}$ provides a measure for the strength of the bouyancy forces.

In the horizontal direction periodic boundary conditions, with spatial period $L$, are applied, while top and bottom planes are assumed as impenetrable, stress-free, and held on constant temperatures:

$$
\begin{aligned}
\left(\frac{\partial v_{x}}{\partial y}\right)_{y=0,1}=v_{y}(x, 0) & =v_{y}(x, 1)=0 \\
\theta(x, 0) & =\theta(x, 1)=0
\end{aligned}
$$

These boundary conditions admit the Fourier expansions

$$
\begin{aligned}
v_{x} & =\sum_{n_{x}=-\infty}^{\infty} \sum_{n_{y}=0}^{\infty} v_{\mathbf{n}}^{(x)} \exp \left(i k_{x} x\right) \cos \left(k_{y} y\right), \\
v_{y} & =\sum_{n_{x}=-\infty}^{\infty} \sum_{n_{y}=1}^{\infty} v_{\mathbf{n}}^{(y)} \exp \left(i k_{x} x\right) \sin \left(k_{y} y\right) \\
\theta & =\sum_{n_{x}=-\infty}^{\infty} \sum_{n_{y}=1}^{\infty} \theta_{\mathbf{n}} \exp \left(i k_{x} x\right) \sin \left(k_{y} y\right) \\
p & =\sum_{n_{x}=-\infty}^{\infty} \sum_{n_{y}=0}^{\infty} p_{\mathbf{n}} \exp \left(i k_{x} x\right) \cos \left(k_{y} y\right)
\end{aligned}
$$

where wavenumbers $\mathbf{k}=\left(k_{x}, k_{y}\right)$ are referred to by indices $\mathbf{n}=\left(n_{x}, n_{y}\right)$ according to

$$
\begin{aligned}
& k_{x}=n_{x} \frac{2 \pi}{L} \quad n_{x}=0, \pm 1, \pm 2, \ldots \\
& k_{y}=n_{y} \pi \quad n_{y}=0,1,2,3, \ldots
\end{aligned}
$$

We use the abbreviations

$$
\mathbf{w}=(\mathbf{v} \cdot \nabla) \mathbf{v}, \quad \phi=\mathbf{v} \cdot \nabla \theta
$$

for the nonlinearities, which have Fourier expansions analogous to $\mathbf{v}$ and $\theta$. In Fourier space Eqs. (1)-(3) then take the form

$$
\begin{aligned}
0 & =i k_{x} v_{\mathbf{n}}^{(x)}+k_{y} v_{\mathbf{n}}^{(y)}, \\
\dot{v}_{\mathbf{n}}^{(x)} & =-w_{\mathbf{n}}^{(x)}-i k_{x} p_{\mathbf{n}}-\mathcal{P} \mathbf{k}^{2} v_{\mathbf{n}}^{(x)}, \\
\dot{v}_{\mathbf{n}}^{(y)} & =-w_{\mathbf{n}}^{(y)}+k_{y} p_{\mathbf{n}}-\mathcal{P} \mathbf{k}^{2} v_{\mathbf{n}}^{(y)}+\mathcal{P} \mathcal{R} \theta_{\mathbf{n}}, \\
\dot{\theta}_{\mathbf{n}} & =-\phi_{\mathbf{n}}+v_{\mathbf{n}}^{(y)}-\mathbf{k}^{2} \theta_{\mathbf{n}} .
\end{aligned}
$$

The pressure $p$ can be eliminated from these equations by taking the divergence of Eq. (2). On observing Eq. (1) this leads to

$$
\nabla \cdot \mathbf{w}=-\triangle p+\mathcal{P} \mathcal{R} \frac{\partial \theta}{\partial y}
$$

which can be solved for $p$ in Fourier space:

$$
p_{\mathbf{k}}=i \frac{k_{x}}{\mathbf{k}^{2}} w_{\mathbf{k}}^{(x)}+\frac{k_{y}}{\mathbf{k}^{2}} w_{\mathbf{k}}^{(y)}-\mathcal{P} \mathcal{R} \frac{k_{y}}{\mathbf{k}^{2}} \theta_{\mathbf{k}} .
$$

Equations (15) and 16) then become

$\dot{v}_{\mathbf{n}}^{(x)}=-\frac{k_{y}^{2}}{\mathbf{k}^{2}} w_{\mathbf{n}}^{(x)}-i \frac{k_{x} k_{y}}{\mathbf{k}^{2}} w_{\mathbf{n}}^{(y)}+\mathcal{P} \mathcal{R} i \frac{k_{x} k_{y}}{\mathbf{k}^{2}} \theta_{\mathbf{n}}$ $-\mathcal{P} \mathbf{k}^{2} v_{\mathbf{n}}^{(x)}$,

$\dot{v}_{\mathbf{n}}^{(y)}=i \frac{k_{x} k_{y}}{\mathbf{k}^{2}} w_{\mathbf{n}}^{(x)}-\frac{k_{x}^{2}}{\mathbf{k}^{2}} w_{\mathbf{n}}^{(y)}+\mathcal{P} \mathcal{R} \frac{k_{x}^{2}}{\mathbf{k}^{2}} \theta_{\mathbf{n}}-\mathcal{P} \mathbf{k}^{2} v_{\mathbf{n}}^{(y)}$.

Because of the condition (14), these two equations are not independent of each other.

From the relation

$$
\boldsymbol{v} \cdot \boldsymbol{\nabla} v_{x}=\frac{\partial}{\partial x} v_{x}^{2}+\frac{\partial}{\partial y}\left(v_{x} v_{y}\right)
$$

it is seen that the spatial mean of $\boldsymbol{v} \cdot \nabla v_{x}$ vanishes (by virtue of the boundary conditions). Thus according to Eqs. (13) and $(15), v_{(0,0)}^{(x)}$ is independent of time. As any mean flow in the $x$ direction can be removed by a Galilean transformation, we have restricted ourselves to the case of $v_{(0,0)}^{(x)}=0$. Our time-asymptotic states are then marginally stable with respect to states with a nonvanishing mean flow.

The system of ordinary differential equations for the Fourier coefficients of velocity and temperature has been studied numerically by means of a pseudospectral method [12,13], mainly with a resolution of $64 \times 32$, but partially (Sec. V) also with a resolution of $128 \times 64$ collocation points.

\section{SYMMETRIES}

With the periodic boundary conditions in the horizontal direction, the domain of the fluid can be considered as a channel of infinite length. In such a channel a largescale horizontal flow is possible $[14,10,9]$. One can split the Fourier expansion of the horizontal velocity according to

$$
\begin{aligned}
v_{x}(x, y, t)= & \sum_{n_{y}=1}^{\infty} v_{\left(0, n_{y}\right)}^{(x)} \cos \left(k_{y} y\right) \\
& +\sum_{\substack{n_{x}=-\infty \\
n_{x} \neq 0}}^{\infty} \sum_{n_{y}=1}^{\infty} v_{\mathbf{n}}^{(x)} \exp \left(i k_{x} x\right) \cos \left(k_{y} y\right) \\
= & \bar{v}_{x}(y, t)+u_{x}(x, y, t) .
\end{aligned}
$$

The large-scale flow $\bar{v}_{x}(y, t)$ is built up by the modes $\left(0, n_{y}\right)$. Each mode of this type corresponds to a shear flow with $n_{y}$ neutral sheets. The modes with odd (even) $n_{y}$ are antisymmetric (symmetric) with respect to the horizontal midline $y=1 / 2$. 
At the onset of convection, immediately after the bifurcations from the quiescent ground state to stationary convection rolls, a descrete subgroup $\mathcal{G}$ of the total symmetry group of the system proves to be important (cf. Refs. $[10,9]) . \mathcal{G}$ consists of four elements,

$$
\mathcal{G}=\left\{i d, S_{1}, S_{2}, S_{3}\right\}
$$

where

$$
\begin{aligned}
S_{1}: & (x, y) \longrightarrow(-x, y), \\
& \left(v_{x}, v_{y}, \theta\right) \longrightarrow\left(-v_{x}, v_{y}, \theta\right) \\
S_{2}: & (x, y) \longrightarrow(-x+L / 2,1-y), \\
& \left(v_{x}, v_{y}, \theta\right) \longrightarrow\left(-v_{x},-v_{y},-\theta\right) \\
S_{3}: & (x, y) \longrightarrow(x+L / 2,1-y), \\
& \left(v_{x}, v_{y}, \theta\right) \longrightarrow\left(v_{x},-v_{y},-\theta\right)
\end{aligned}
$$

$S_{1}$ is a reflection in the $y$ axis (or the vertical midline); $S_{2}$ corresponds to a rotation by 180 degree about the point $(0,1 / 2)$ plus a horizontal translation by $L / 2 ; S_{3}=S_{1} \circ S_{2}$ is the product of these two transformations, which is equivalent to a reflection in the horizontal midline combined with a horizontal translation by $L / 2$. The group is isomorphic to the dihedral group $D_{2}$ [15]. It is commutative and each transformation gives the identity if it is applied twice.

With the primary bifurcation from the conductive state convection sets in in the form of stationary rolls symmetric with respect to the full group $\mathcal{G}$. For the next bifurcation, if it is symmetry breaking, there are, with the exception of the trivial symmetry group $\{i d\}$, only three possibilities to get a subgroup with respect to which a bifurcating solution can be symmetric:

$$
\begin{aligned}
& \text { 1. } \mathcal{G}_{1}=\left\{i d, S_{1}\right\} \\
& \text { 2. } \mathcal{G}_{2}=\left\{i d, S_{2}\right\} \\
& \text { 3. } \mathcal{G}_{3}=\left\{i d, S_{3}\right\}
\end{aligned}
$$

Each of these three possibilities has a particular consequence for the large-scale flow $\bar{v}_{x}(y, t)$. In case 1 , because of $\bar{v}_{x}(y, t)=-\bar{v}_{x}(y, t)$, the large-scale flow has to be zero. In case 2 one finds $\bar{v}_{x}(y, t)=-\bar{v}_{x}(1-y, t)$, i.e., the largescale flow has to be antisymmetric to the line $y=1 / 2$. Case 3 , finally, corresponds to a large-scale flow symmetric to the line $y=1 / 2: \bar{v}_{x}(y, t)=\bar{v}_{x}(1-y, t)$.

The symmetries have also particular signatures in Fourier space. For $S_{1}$ we have the following symmetries for the Fourier coefficients:

$$
\begin{aligned}
& v_{\left(n_{x}, n_{y}\right)}^{(x)}=-v_{\left(-n_{x}, n_{y}\right)}^{(x)} \\
& v_{\left(n_{x}, n_{y}\right)}^{(y)}=v_{\left(-n_{x}, n_{y}\right)}^{(y)} \\
& \theta_{\left(n_{x}, n_{y}\right)}=\theta_{\left(-n_{x}, n_{y}\right)}
\end{aligned}
$$

The coefficients $v_{\left(0, n_{y}\right)}^{(x)}$, corresponding to a large-scale flow, have to be zero. For $S_{2}$ one finds

$$
\begin{aligned}
& v_{\left(n_{x}, n_{y}\right)}^{(x)}=(-1)^{n_{x}+n_{y}+1} v_{\left(-n_{x}, n_{y}\right)}^{(x)} \\
& v_{\left(n_{x}, n_{y}\right)}^{(y)}=(-1)^{n_{x}+n_{y}} v_{\left(-n_{x}, n_{y}\right)}^{(y)} \\
& \theta_{\left(n_{x}, n_{y}\right)}=(-1)^{n_{x}+n_{y}} \theta_{\left(-n_{x}, n_{y}\right)}
\end{aligned}
$$

We call the modes $\left(n_{x}, n_{y}\right)$ even when $n_{x}+n_{y}$ is even and odd when $n_{x}+n_{y}$ is odd. For the last symmetry, $S_{3}$, one has

$$
\begin{array}{r}
v_{\left(n_{x}, n_{y}\right)}^{(x)}=v_{\left(n_{x}, n_{y}\right)}^{(y)}=\theta_{\left(n_{x}, n_{y}\right)}=0, \\
\text { if } n_{x}+n_{y} \text { is odd, }
\end{array}
$$

i.e., all odd modes vanish.

Explicitly imposing the symmetries $S_{1}$ and $S_{3}$, which generate the group $\mathcal{G}$, Curry et al. [8] studied solutions with the full symmetry. By this restriction to the invariant subspace of the fully symmmetric solutions, symmetry-breaking bifurcations were suppressed and, in particular, large-scale flows excluded. For the aspect ratio $L=2 \sqrt{2}$ and the Prandtl number $\mathcal{P}=6.8$, Curry et al. found a transition from stationary to periodic convection at a Rayleigh number of $\tilde{\mathcal{R}}_{1} \approx 90 \mathcal{R}_{c} \approx 59200$ and a transition from periodic to quasiperiodic convection involving two frequencies at a Rayleigh number of $\tilde{\mathcal{R}}_{2} \approx$ $290 \mathcal{R}_{c} \approx 190700$. $\mathcal{R}_{c}=27 \pi^{4} / 4=657.5$ is the critical Rayleigh number at which stationary convection sets in. At very high Rayleigh number $\left(\tilde{\mathcal{R}}_{3} \approx 800 \mathcal{R}_{c} \approx 526000\right)$, Curry et al. then observed a transition back to periodic convection with one frequency. In a control computation without symmetries imposed they found the first transition to periodic convection to take place at a Rayleigh number of $\mathcal{R}_{1} \approx 50 \mathcal{R}_{c} \approx 32800$.

\section{BIFURCATIONS TO LIMIT CYCLES AND 2-TORI; LARGE-SCALE FLOW}

To have a direct comparison, we assume the same aspect ratio and the same Prandtl number as used in Ref. [8], namely, $L=2 \sqrt{2}$ and $\mathcal{P}=6.8$. The first bifurcation from stationary convection rolls takes place between Rayleigh numbers $30000=45.6 \mathcal{R}_{c}$ and $31000=47.1 \mathcal{R}_{c}$; it is a transition from stationary to periodic convection. This agrees with the result of Ref. [8] mentioned in Sec. III. The bifurcating periodic solution, if looked upon at one instant of time, has the spatial symmetry $S_{1}$, while the symmetries $S_{2}$ and $S_{3}$ are broken. A large-scale flow does not arise because $S_{1}$ is preserved.

In Fig. 1 velocity streamlines are shown for four instants of time within one oscillation period $T_{1}$. One can see that the convection rolls undergoe a periodic deformation but remain mirror symmetric to the vertical line $x=L / 2$. The streamline profiles at $t=0$ and $t=T_{1} / 2$ $\left(t=T_{1} / 4\right.$ and $\left.t=3 T_{1} / 4\right)$ can be transformed into each other by applying $S_{2}$ or $S_{3}$. This observation leads to a more precise characterization of the symmetry. Actually the periodic orbit as a whole is invariant also with 
respect to $S_{2}$ and $S_{3}$. By the action of these transformations merely a time shift is produced. As each of the transformations is inverse to itself, the time shift must be just half a period. A periodic state with the same type of symmetry as the result of the first bifurcation from stationary convection was found by Prat et al. [9] for the case of no-slip boundary conditions, an aspect ratio of $L=2$, and a Prandtl number of $\mathcal{P}=10$.

The next bifurcation, taking place between $\mathcal{R}=$ $45000=68.4 \mathcal{R}_{c}$ and $\mathcal{R}=46000=70.0 \mathcal{R}_{c}$, is a transition from periodic to quasiperidic convection. In this bifurcation also the spatial mirror symmetry $S_{1}$ is broken and a large-scale flow arises. Figs. 2 and 3 confirm that now two frequencies are involved. Fig. 2 shows a two-dimensional projection of the trajectory in the highdimensional space of modes. Obviously, the motion takes place on a 2-torus with two incommensurable frequencies. Now the three symmtries are completely broken. If one of the transformations $S_{1}, S_{2}, S_{3}$ would merely time shift a solution, this solution would be transformed into itself by applying the transformation (the time shift) twice, which is possible only for time-periodic solutions. So there coexist four different solutions. Given one of them, the three others are obtained by applying the transformations $S_{1}$, $S_{2}, S_{3}$.

In Fig. 3 the power spectrum of the time evolution of an odd mode is shown. The two basic frequencies are $f_{1}=86$ and $f_{2}=17 . f_{2}$ is the new frequency, which is the strongest frequency in the power spectra of the odd modes $\left(0, n_{y}\right)$ (not shown) corresponding to largescale flow. In the torus bifurcation both the odd and the even modes $\left(0, n_{y}\right)$ are excited. Very often the presence of an antisymmetric shear motion, corresponding to odd modes, is considered as responsible for a tilting of the convection rolls (or of the lines separating different rolls, respectively). We would like to note here that the even modes $\left(0, n_{y}\right)$ lead to a tilting, while the odd modes enhance one roll (say, the clockwise) and suppress the other (counter-clockwise). Namely, a shear motion, to the right at the top and to the left at the bottom, enhances clockwise motion - the corresponding roll becomes largerand impedes counter-clockwise motion - the corresponding roll becomes smaller. No tilting is produced. By the motion corresponding to even modes, on the other hand, one roll is enhanced at the top and suppressed at the bottom, while the other is suppressed at the top and enhanced at the bottom. As a result, the rolls begin to resemble triangles, one with top up, the other with top down, and the line separating them is tilted.

A periodic tilting can be seen in Fig. 4, which shows stream profiles for $t=0, t=T_{2} / 8, t=T_{2} / 4$ and $t=3 T_{2} / 8$ (a triangle-like deformation, though very weak, is also recognizable). In the first plot the 'vertical' line separating two convection cells is tilted slightly to the right, in the next plot to the left, then in the following again to the right, and so on. The rolls are tilted to the left and to the right approximately four times in each period $T_{2}$. The explanation of this can be seen in Fig. 5, which shows the large-scale flow $\bar{v}_{x}(y, t)$ (abscissa) versus $y$ (ordinate) for nine instants of time. The first plot shows all modes, the second only odd modes and the third only even modes. At some instants of time the total flow is approximately symmetric, at others approximately antisymmetric to the horizontal midline. The plot of the odd modes is dominated by the first odd mode, $(0,1)$, and the plot of the even modes by the first even mode, $(0,2)$. One sees that the even modes are changing approximately four times as fast as the odd modes. The strongest frequency for even modes is $f_{1}-f_{2}$, which is close to $4 f_{2}$. It is clearly the influence of the even modes that leads to the tilting seen in Fig. 4.

\section{BIFURCATIONS TO 3- AND 4-TORI; PHASE LOCKINGS}

We have traced the torus branch for increasing values of the Rayleigh number. At $\mathcal{R} \approx 85000$ a third frequency appears. This frequency corresponds to directionreversing traveling waves [16], namely, the whole oscillating structure drifts, very slowly, forth and back in the $x$ direction. The time between the direction reversals is of the order of the thermal diffusion time $-f_{3} \approx 3 / 4$ and time is measured in units of the thermal diffusion time. The modes $\left(0, n_{y}\right)$, corresponding to the large-scale flow, remain unaffected by the third frequency, whereas all other modes now show three superposed oscillations.

The degenerate 3-torus (degenerate because some modes show only two frequencies) determines the dynamics over a large interval of the Rayleigh number, up to a value of about 200000 . There are two subintervals, between $\mathcal{R} \approx 90000$ and $\mathcal{R} \approx 95000$ as well as between $\mathcal{R} \approx 175000$ and $\mathcal{R} \approx 180000$, in which the two frequencies corresponding to real oscillations (the first one and the second one) are locked, so that the degenerate 3torus becomes a degenerate 2-torus. Interestingly, during the phase lockings the traveling waves are nonreversing, i.e., the oscillating structure migrates in one direction. The character of these phase-locked solutions is demonstrated in Figs. 6 a), b). In Fig. 6a) the trajectory is projected onto the plane spanned by two 'normal' components, namely the real and imaginary parts of $v_{(5,1)}^{(x)}$. Two incommensurable frequencies are involved. The traveling wave appears as a very slow circular rotation. In Fig. 6b), showing a projection onto modes $\left(0, n_{y}\right)$, the frequency of the traveling wave is absent and the orbit thus closed.

We have traced this branch up to $\mathcal{R}=230000$, expecting a transition to chaos. Indeed, as is seen in Figs. 6c) - f), the motion in phase space becomes more and more irregular, suggestive of a transition to chaos via torus destruction $[17,18]$. Also the frequency spectrum has now the broadband character of chaotic solutions. To test for chaos, we have calculated the largest Lyapunov exponents, using an algorithm of Shimada and Nagashima [19]. Fig. 7 shows for $\mathcal{R}=230000$ the cumulative values 
of the 10 largest Lyapunov exponents versus the integration time. Surprisingly, five of the exponents tend to zero and no exponent tends to a positive limit. Four exponents approach zero from above (two of them are very close to each other and are seen to be different only in enlarged figures). That these four exponents do not tend to positive values, but approach zero approximately following a power law, can be seen from a log-log plot inside Fig. 7 (as the logarithm is not defined for negative arguments, negative exponents are absent in the log-log plot). From the plots in Fig. 7 we conclude that the attractor is a 4-torus. The fifth vanishing Lyapunov exponent corresponds to the translational symmetry of the system in the $x$ direction, which appears as a direction of marginal stability in phase space.

Besides the solutions described so far, we have detected a coexisting solution branch. Between $\mathcal{R} \approx 140000$ and $\mathcal{R} \approx 180000$ the solutions of this branch are normal (unidirectional) modulated traveling waves, i.e., lie on a degenerate 2 -torus. The modes $\left(0, n_{y}\right)$ oscillate with just one frequency (the frequency of the modulation). Between $\mathcal{R} \approx 180000$ and $\mathcal{R} \approx 230000$ the solutions are simply periodic, seemingly as the result of a phase locking on the 2-torus, and belong to the symmetry group $\mathcal{G}_{3}$ described in Sec. III. When traced to smaller Rayleigh numbers, this branch disappears for $\mathcal{R} \approx 140000$. Its basin of attraction seems to be very small and we could not clarify its origin.

\section{DISCUSSION}

We have studied the bifurcations in two-dimensional Rayleigh-Bénard convection with stress-free boundary conditions and no symmetry imposed. As expected, the bifurcation sequence differs significantly from that found by Curry et al. [8] for the case with symmetries imposed. The first bifurcation following the primary one (that from the quiescent ground state to stationary symmetric convection rolls) is a Hopf bifurcation at a Rayleigh number of $R \approx 30000$, leading to simple oscillations for which, at a given instant of time, velocity and temperature are still mirror symmetric to the vertical midline $x=L / 2$. Then in a second Hopf bifurcation at $\mathcal{R} \approx 45000$, leading to a torus solution with two incommensurable frequencies, also this remaining symmetry is broken. The torus solution shows a large-scale (i.e., independent of $x$ ) horizontal flow component, the vertical profile of which is neither symmetric nor antisymmetric to the horizontal midline $y=1 / 2$. Contrary to a prevalent belief, it is its symmetric, rather than its antisymmetric part that causes a periodic tilting of the convection rolls.

For further increased Rayleigh number then traveling waves appear and the solution undergoes phase lockings. By the appearance of traveling waves at $R \approx 85000$, the 2 -torus bifurcates to a degenerate 3 -torus. The modes $\left(0, n_{y}\right)$, corresponding to the large-scale flow, remain un- affected by this bifurcation (i.e., show only two frequencies), whereas all other modes now exhibit three superposed oscillations. Interestingly, the traveling waves are direction-reversing in general and unidirectional in the intervals of the phase lockings.

Up to $\mathcal{R}=230000$ we did not find chaos (this agrees with the results of Curry et al. [8]), though the motion in phase space becomes more and more irregular. The calculation of the largest Lyapunov exponents for $\mathcal{R}=$ 230000 indicates that the system moves on a 4-torus.

Now according to a theorem by Ruelle, Takens, and Newhouse $[17,20]$, bifurcations from 2-tori lead generically to chaotic attractors. It is not clear yet, however, whether the hydrodynamic equations (general, three-dimensional) are generic in the required sense. If not, nonchaotic attracting motions on three- or higherdimensional tori might well be observable. The laboratory experiments on convection do not give a clear picture in this respect. On one hand there are experiments showing the sequence stationary $\rightarrow$ simply periodic $\rightarrow$ two-frequency quasiperiodic $\rightarrow$ chaotic [21]. On the other hand also counterexamples, namely quasiperiodic states with three incommensurable frequencies (3-tori), were observed, for instance by Gollub and Benson [22], who found the scenario for the transition to nonperiodic or turbulent states to depend in an intricate way on the system parameters.

Seemingly the equations of two-dimensional Boussinesq convection (with stress-free vertical and periodic horizontal boundary conditions) are nongeneric in the sense of the Ruelle-Takens-Newhouse theorem. It may now be that any small perturbation towards threedimensionality destroys the behavior found. But it is also conceivable that systems in an appropriately defined neighborhood of the studied one still behave in a qualitatively similar way.

Some remarks concerning the physical realizability of two-dimensional convection seem to be in order. As reviewed in Ref. [23] (p. 245), two-dimensionality of the motion can be experimentally realized by confining the fluid between parallel (in our case, vertical) plane walls sufficiently close to each other. If these walls are electrically insulating and the fluid is electrically conducting (e.g., an electrolytic solution), disturbing boundary-layer effects may be largely suppressed by applying a uniform magnetic field perpendicular to the walls. Such a magnetic field impedes motions with gradients in the direction of the field. The ensuing two-dimensional motion satisfies the equations for nonconducting fluids (see Ref. [24], p. 298).

Both the two-dimensionality of the motion and the periodic horizontal boundary conditions can be approximately realized in convection experiments in the geometry of narrow cylindrical annuli with the cylinder axis in the vertical direction (parallel to the directions of gravity and the applied temperature gradient). Such experiments have been reported, for instance, by Ciliberto [25] and by Daviaud and Dubois [26], but the corresponding 
aspect ratios (i.e., numbers of parallel rolls) are probably much too large to allow for a direct comparison with our calculations. A similar annulus experiment, with stressfree top and bottom boundaries but the convection driven by the combined effects of buoyancy and surface tension, has been described by Bensimon [27].

An alternative possibility are rapidly rotating annuli with the temperature gradient in the radial direction and the convection driven by centrifugal buoyancy. Such systems have been studied both theoretically and experimentally (cf. Ref. [28]), among other things in order to model the zonal flows in the atmospheres of the major planets - these zonal flows correspond to the large-scale horizontal flows of the present study. A potential further application of this kind of annulus model is the differential rotation of the sun [29] (here the convection is again mainly driven by gravitational buoyancy). The rotation gives an effective stiffness against variations along the rotational axis, thus admitting a theoretical description of basic dynamical phenomena by the equations of twodimensional convection.

In rectangular containers convection typically sets in in the purely two-dimensional form of straight rolls whose axes are parallel to the shorter side of the container [5]; in the case of narrow cylindrical annuli with the buoyancy forces parallel to the axis of the cylinder, the roll axes are radially oriented, while they are parallel to the cylinder axis for the rapidly rotating annuli. If the experimental conditions are chosen such that, perhaps to a varying extent, two-dimensional motions are favored, bifurcations like those studied in this paper (including possibly such to nonchaotic higher-dimensional torus solutions), which leave the motion two-dimensional, might occur before other types of bifurcations eventually lead to three-dimensional convection.

Due to numerical limitations, the solutions could not be traced into the region of $\mathcal{R}>230000$. So it remains an open question whether at higher Rayleigh numbers chaos is still reached. This problem, as well as the possible changes of the bifurcation scenario if a larger aspect ratio is used, will be the subject of future studies with improved numerics.

[1] S. Chandrasekhar, Hydrodynamic and Hydromagnetic Stability (Clarendon Press, Oxford, 1961).

[2] A. Schlüter, D. Lortz, and F. H. Busse, J. Fluid Mech. 23, 129 (1965).

[3] T. G. Drazin and W. H. Reid, Hydrodynamic Stability (Cambridge University Press, Cambridge, England, 1981).

[4] F. H. Busse, in Hydrodynamic Instabilities and the Transition to Turbulence, Vol. 45 of Topics in Applied Physics, edited by H. L. Swinney and J. P. Gollub (Springer,
Berlin, Heidelberg, 1985), pp. 97-133.

[5] E. L. Koschmieder, Bénard Cells and Taylor Vortices (Cambridge University Press, Cambridge, England, 1993).

[6] E. N. Lorenz, J. Atmos. Sci. 20, 130 (1963).

[7] J. H. Curry, Commun. Math. Phys. 60, 193 (1978).

[8] J. H. Curry, J. R. Herring, J. Loncaric, and S. A. Orszag, J. Fluid Mech. 147, 1 (1984).

[9] J. Prat, J. M. Massaguer, and I. Mercader, Phys. Fluids 7, 121 (1995).

[10] J. Prat, I. Mercader, and J. M. Massaguer, Int. J. Bifurcation \& Chaos 4, 1369 (1994).

[11] R. J. Goldstein and D. J. Graham, Phys. Fluids 12, 1133 (1969).

[12] C. Canuto, M. Y. Hussaini, A. Quarteroni, and T. A. Zang, Spectral Methods in Fluid Dynamics (Springer, Berlin, 1988).

[13] J. P. Boyd, Chebyshev and Fourier Spectral Methods (Springer, Berlin, 1989).

[14] L. N. Howard and R. Krishnamurti, J. Fluid Mech. 170, 385 (1986).

[15] M. Golubitsky, I. Stewart, and D. G. Schaeffer, Singularities and Groups in Bifurcation Theory (Springer, New York, 1988), Vol. II.

[16] A. S. Landsberg and E. Knobloch, Phys. Lett. A 159, 17 (1991).

[17] S. Newhouse, D. Ruelle, and F. Takens, Commun. Math. Phys. 64, 35 (1978).

[18] V. Franceschini and C. Tebaldi, Commun. Math. Phys. 94, 317 (1984).

[19] I. Shimada and T. Nagashima, Progr. Theor. Phys. 61, 1605 (1979).

[20] D. Ruelle and F. Takens, Commun. Math. Phys. 20, 167 (1971).

[21] H. L. Swinney and J. P. Gollub, Phys. Today 31, 41 (1978).

[22] J. P. Gollub and S. V. Benson, J. Fluid Mech. 100, 449 (1980).

[23] M. Lesieur, Turbulence in Fluids (Kluwer, Dordrecht, 1990).

[24] R. Moreau, Magnetohydrodynamics (Kluwer, Dordrecht, 1990).

[25] S. Ciliberto, Eur. J. Mech. B/Fluids 10, 193 (1991).

[26] F. Daviaud and M. Dubois, Eur. J. Mech. B/Fluids 10, 199 (1991).

[27] D. Bensimon, Phys. Rev. A 37, 200 (1988).

[28] F. H. Busse, Chaos 4, 123 (1994).

[29] G. Rüdiger, Differential Rotation and Stellar Convection: Sun and Solar-type Stars (Akademie-Verlag, Berlin, 1989). 

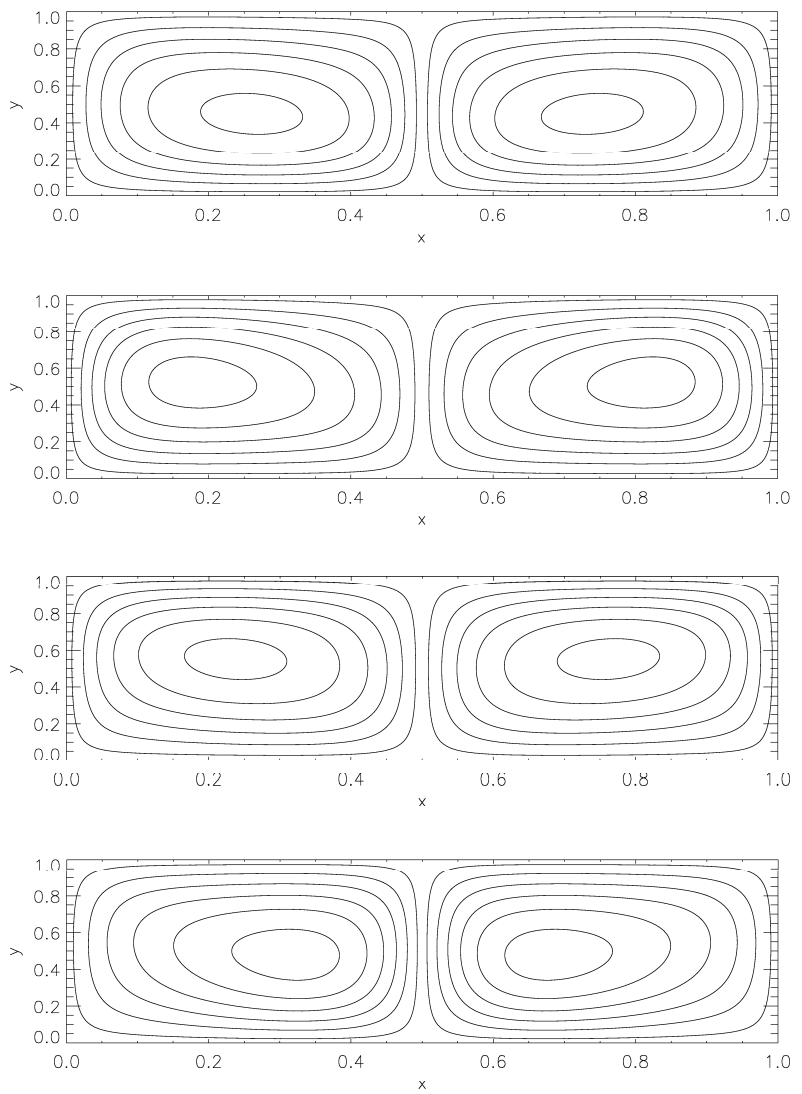

FIG. 1. Streamlines at $\mathcal{R}=40000=60.8 \mathcal{R}_{c}$ for $t=0$, $t=T_{1} / 4, t=T_{1} / 2$ and $t=3 T_{1} / 4$.

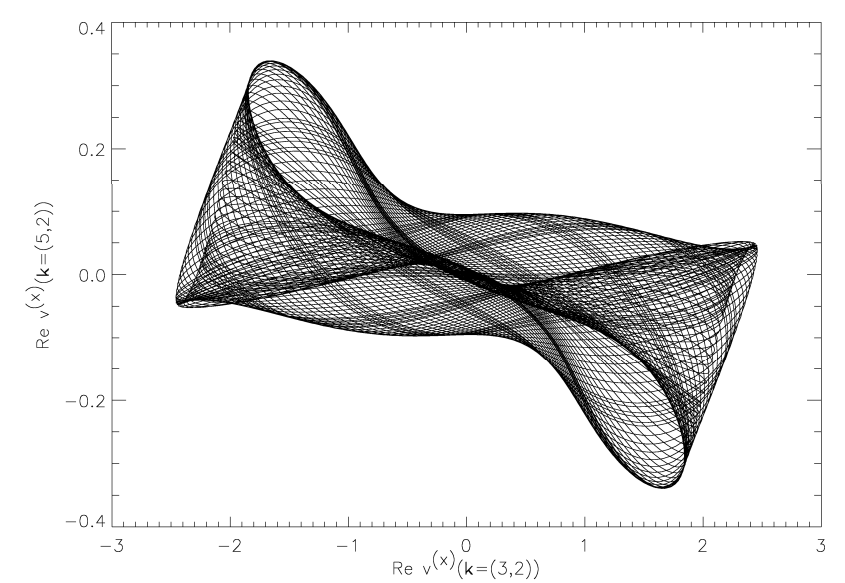

FIG. 2. Phase space projection to the plane spanned by $\operatorname{Re}\left(v_{(3,2)}^{(x)}\right)$ and $\operatorname{Re}\left(v_{(5,2)}^{(x)}\right)$ at $\mathcal{R}=46000=70.0 \mathcal{R}_{c}$.

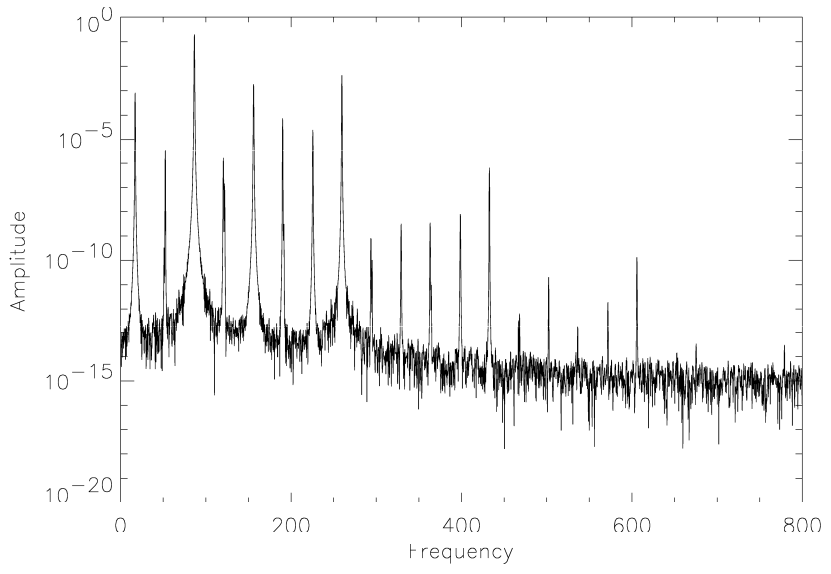

FIG. 3. Frequency spectrum of the time evolution of $\operatorname{Re}\left(v_{(3,2)}^{(x)}\right)$ at $\mathcal{R}=46000=70.0 \mathcal{R}_{c}$.
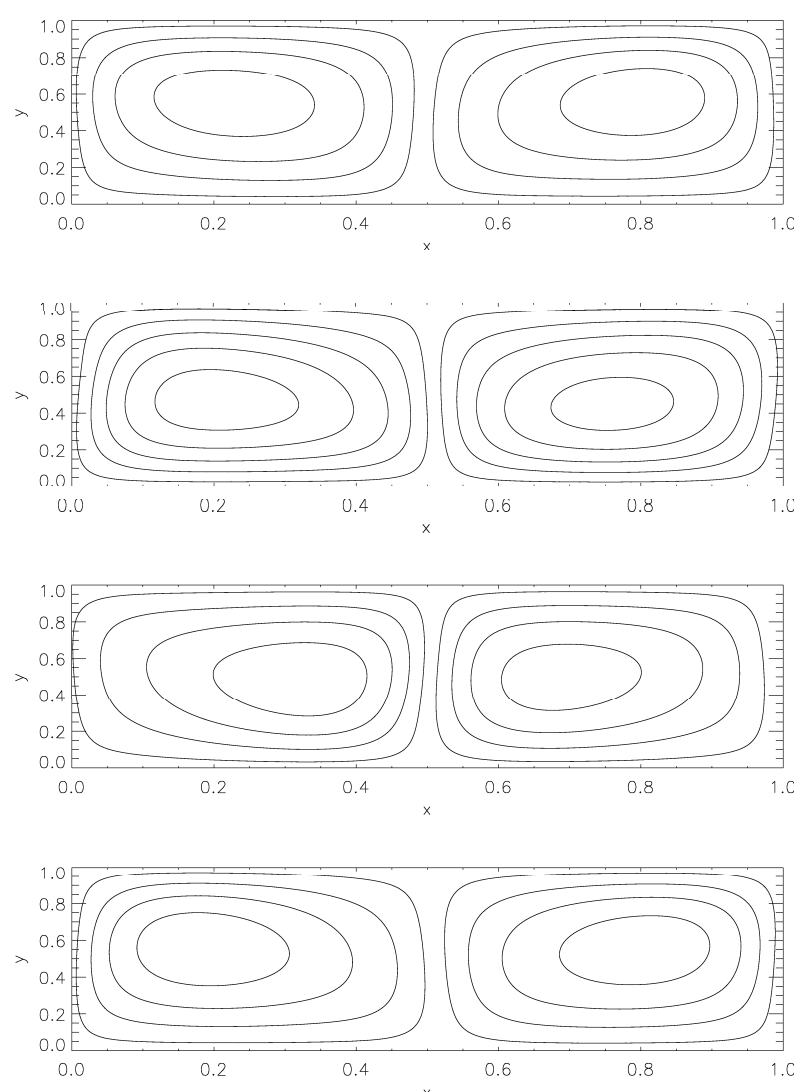

FIG. 4. Streamlines at $\mathcal{R}=50000=76.0 \mathcal{R}_{c}$ for $t=0$, $t=T_{2} / 8, t=T_{2} / 4$ and $t=3 T_{2} / 8$. 

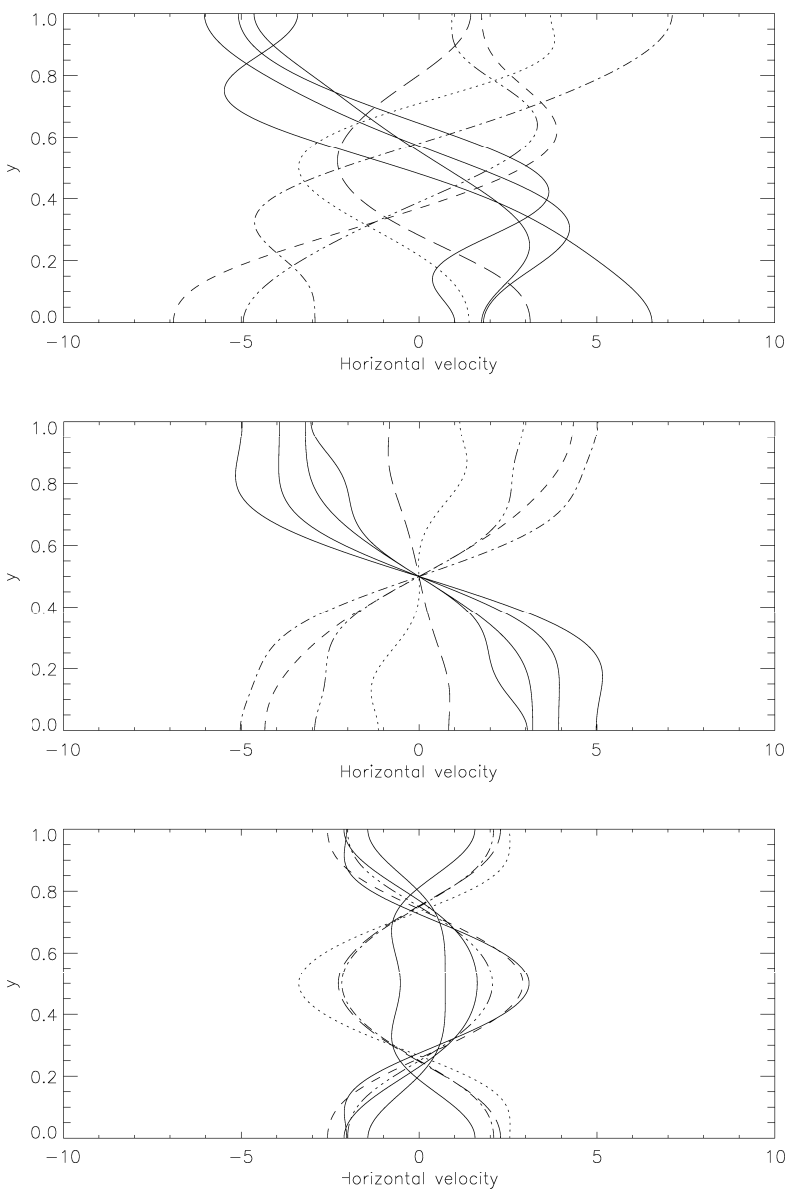

FIG. 5. Large-scale flow $\bar{v}_{x}(y)$ at $\mathcal{R}=50000=76.0 \mathcal{R}_{c}$ (abscissa) versus $y$ (ordinate). The first plot shows all modes, the second only the odd modes, and the third only the even modes. The flow is shown for $t=0$ (solid line), $t=T_{2} / 8$ (dots), $t=2 T_{2} / 8$ (short dashes), $t=3 T_{2} / 8$ (one dash, one dot), $t=4 T_{2} / 8$ (one dash, three dots), $t=5 T_{2} / 8$ (long dashes), $t=6 T_{2} / 8$ (solid line), $t=7 T_{2} / 8$ (solid line), $t=8 T_{2} / 8$ (solid line).
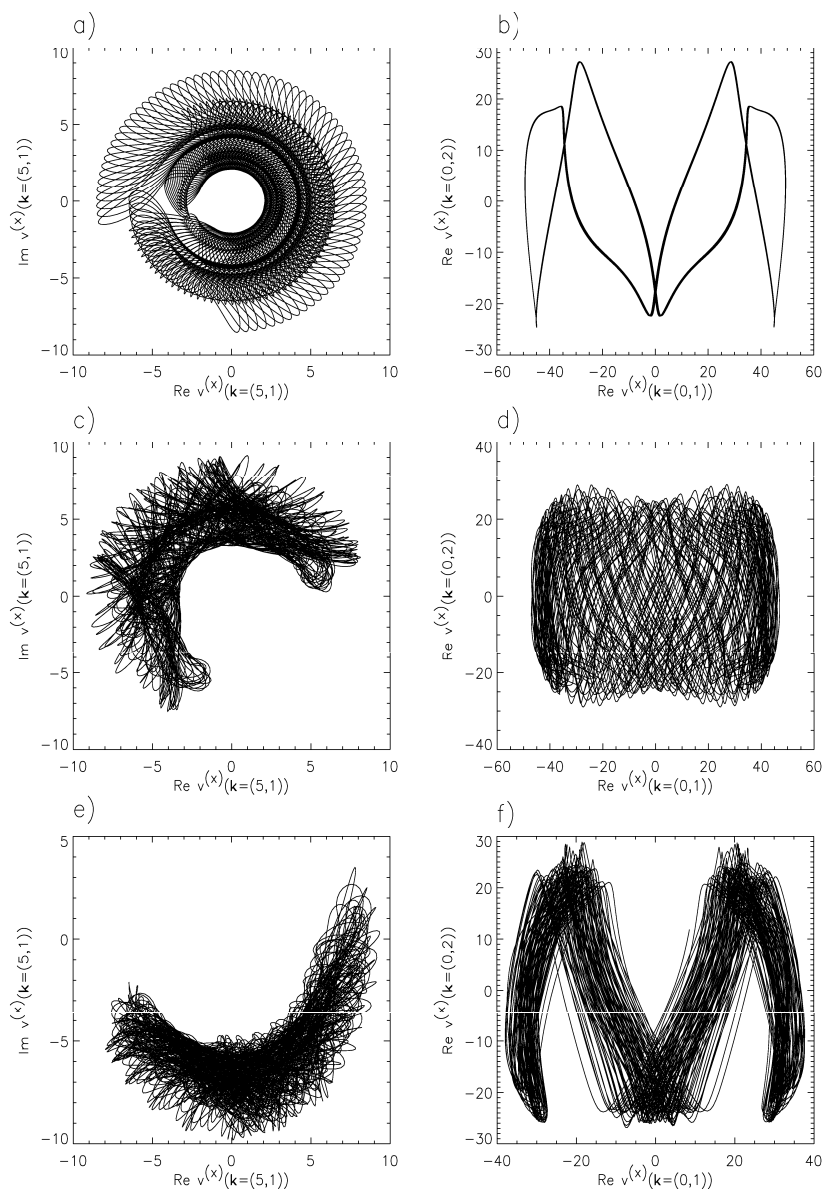

FIG. 6. Phase space projection onto the planes spanned by $\operatorname{Re}\left(v_{(5,1)}^{(x)}\right), \operatorname{Im}\left(v_{(5,1)}^{(x)}\right)$ (left) and by $\operatorname{Re}\left(v_{(0,1)}^{(x)}\right), \operatorname{Re}\left(v_{(0,2)}^{(x)}\right)$ (right) for $\mathcal{R}=175000[\mathrm{a}), \mathrm{b})], \mathcal{R}=200000[\mathrm{c}$ ), d)] and $\mathcal{R}=230000[\mathrm{e}), \mathrm{f})]$.

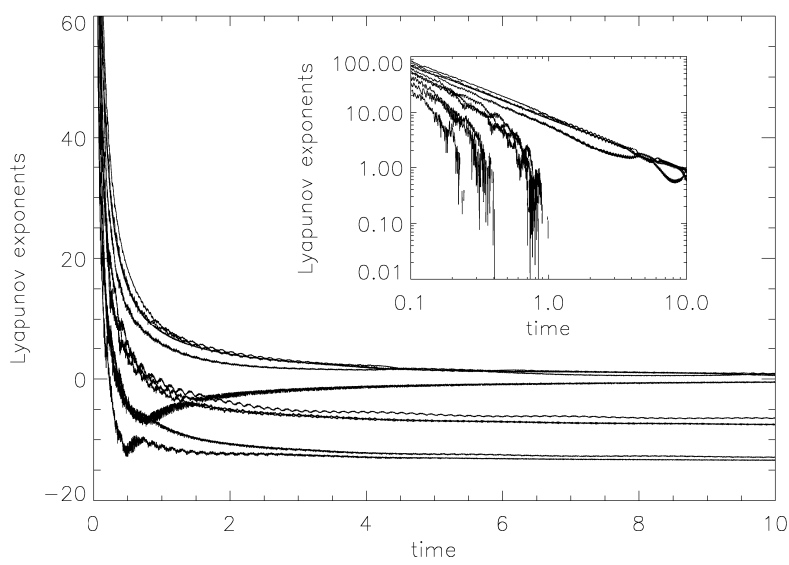

FIG. 7. The 10 largest Lyapunov exponents versus integretion time for $\mathcal{R}=230000$. The inner plot is doubly logarithmic and negative values of the exponents are thus not drawn. 